\title{
Does Diuretic During Extra-Corporeal Shock Wave Lithotripsy (ESWL) Really Help in Stone Fragmentation and Clearance?
}

Dr. Vipin Chandra ${ }^{1 *}$, Rajesh Tiwari ${ }^{2}$

\author{
${ }^{1}$ Assistant Professor, Department of Urology, AIIMS, Patna - Aurangabad Rd, Phulwari Sharif, Patna, Bihar 801507, India \\ ${ }^{2}$ Professor and Head, Department of Urology, Indira Gandhi Institute of Medical Sciences (IGIMS), Allahabad bank, Bailey Rd, \\ Sheikhpura, Patna, Bihar 800014, India
}

DOI: $10.36347 /$ sjams.2020.v08i12.024

| Received: 14.12.2020 | Accepted: 22.12.2020 | Published: 24.12.2020

*Corresponding author: Dr. Vipin Chandra

\section{Abstract}

Original Research Article

Background: Extra-corporeal shock wave lithotripsy (ESWL) is the important mode of stone management for the patients who are fulfilling the criteria for fragmentation and clearance using shock waves. ESWL only helps in fragmentation. Clearance is done by the diuresis. In this study we aim to compare stone clearance with ESWL procedure using diuretics and fluids versus fluid alone. Material and methods: This is a prospective parallel arm randomized open label single center study conducted at Indira Gandhi institute of medical sciences (IGIMS), Patna between January 2019 to December 2019. Inclusion: Patients between 18 to 60 years of age with solitary renal stone of size less than $1.5 \mathrm{~cm}$ measured on ultrasound. Any patient having coagulopathy, positive urine culture, altered anatomy of urinary system etc. are excluded from the study. Enrolled patients were randomized to either standard group or diuretic group. Diuretic group received diuretic in the form of injection furosemide $20 \mathrm{mg}$ slow iv along with continuous $0.9 \%$ Normal Saline (NS) during the procedure (total 1 liter) while standard group received only $0.9 \%$ NS infusion during the procedure. Outcome: Clearance was measured as residual stone after 3 months of last session of ESWL using X ray, ultrasound and NCCT KUB. Result: 130 patients were screened and 94 were found eligible for randomization. Baseline characteristics were similar in both groups. Average size of stone was $1.75 \pm 0.2 \mathrm{~cm}$ in the standard group and $1.64 \pm 0.3$ in the diuretic group. In our present study, the stone clearance rate was significantly better in diuretic group as compared to standard group $(82.97 \%$ vs $59.57 \% \mathrm{P}<0.05)$. Conclusion: We conclude that there is a beneficial role of using diuretics along with hydration in renal stone fragmentation and clearance as compared with hydration alone.

Keywords: Diuretic, ESWL, Renal stone.

Copyright $\odot 2020$ The Author(s): This is an open-access article distributed under the terms of the Creative Commons Attribution 4.0 International License (CC BY-NC 4.0) which permits unrestricted use, distribution, and reproduction in any medium for non-commercial use provided the original author and source are credited.

\section{INTRODUCTION}

Renal stone is one of the important urological condition, incidence varies widely in India due to geographical diversity and variation in food pattern. Clinical presentation is also variable from asymptomatic to stone related obstructive nephropathy. Symptom depends upon the size, location and type of stone. About $12 \%$ of the population is likely to experience urinary stone disease during their lifetime and the recurrence can occur in upto 50\% [1]. In India mostly calcium oxalate and apatite stones are prevalent [2]. Available options for treatment of renal stone disease include: extracorporeal shockwave lithotripsy (ESWL), percutaneous nephrolithotomy (PCNL), Ureterorenoscopy and lithotripsy (URSL), medical expulsive therapy or combination of these. Treatment depends upon stone size, location and composition. ESWL is minimally invasive mode of stone fragmentation. It is well accepted mode of treatment for both adult and pediatric patients. Stone fragmentation and clearance depends upon stone size, location, composition, Hounsfield unit (HU) and built of the patient. Hydration and diuresis may promote the fragmentation and clearance of stone from the pelvicalyceal system (PCS) [3].

Patients with renal stone disease who are planned for the ESWL procedure are usually in a state of dehydration due to use of purgative and fasting for few hours before the procedure to improve the visibility of stone on C-arm/ fluoroscopy. Dehydration causes relative oliguria which may have a negative effect on stone fragmentation due to reduced fluid film surrounding the stone [4]. In addition, reduced renal function due to obstruction by stone may reduce the fluid surrounding the stone in the pelvicalyceal system and also contribute to inadequate fragment clearance. 
To augment stone clearance whether diuretics should be used or not has not been specified in standard recommendations. Previous studies suggest benefit of diuretics but the quality of evidence is not strong enough to prove definitive role of diuretics in ESWL. Hence we planned this randomized controlled trial to assess the benefit of diuretics in stone fragmentation and clearance by ESWL.

\section{Material And Methods}

This is a randomized parallel group open label single centre trial conducted at Indira Gandhi institute of medical sciences (IGIMS), Patna between January 2019 to December 2019. Inclusion criteria: All adults above 18 years of age belonging to either gender with solitary stone of size less than $1.5 \mathrm{~cm}$, located in upper and middle calyx and $\mathrm{HU}<1000$ as measured by combined ultrasonography (USG), intravenous urography (IVU) and CT scan.

\section{Exclusion Criteria}

Patient with anatomical abnormalities like scoliosis, kyphosis, ectopic kidney, duplex moiety, fusion anomalies and ureterocele; coagulopathy; obesity (skin stone distance $>10 \mathrm{~cm}$ ); documented urinary tract infection; previous history of urinary tract surgery; patients with solitary functioning kidney; patients with reduced renal function (serum creatinine $>1.5 \mathrm{mg} / \mathrm{dl}$ ); pregnant women and patients with known severe cardio-pulmonary disease were excluded from the study.

Data of the subjects like detailed history, physical examination, basic laboratory investigations like complete blood count, serum creatinine, urine examination and culture sensitivity were recorded at baseline. For imaging we did ultrasound of kidney ureter and bladder (USG KUB), X-ray/intravenous pyelography (IVP) and non-contrast computed tomography of kidney, ureter and bladder (NCCT KUB) in all patients. Urine culture was done in all cases. Those with urinary tract infection, were treated with antibiotics and repeat culture was done after 1 week. They were included only if urine culture was sterile. Stone profile in terms of size location and density, number based on CT report was recorded. ESWL procedure related details like number of sessions with number of shocks given, any complication was recorded. Final stone burden after three months of last session were recorded on the basis of Xray KUB, USG KUB and NCCT KUB.

\section{Sample Size}

Presuming a difference of $30 \%$ based on previous studies, and targeting a power oof $80 \%$ a minimum sample size of 36 per group was decided. Randomization was done using computer generated table of random numbers. Patients were allocated to either standard group or diuretic group. Diuretic group received diuretic in the form of injection furosemide 20 mg slow iv along with continuous $0.9 \%$ Normal Saline (NS) during the procedure (Total 1 liter) while standard group received only $0.9 \% \mathrm{NS}$ infusion during the procedure. A standard ESWL protocol was used for all the patients. Continuous blood pressure was monitored for all patients during the procedure.

In our institute we use Dornier Compact Sigma ESWL machine with manual operation having both ultrasonography and fluoroscopic as a stone localizer. In each session we have given 2500 shocks at the rate of 60 shocks per minute, with gradual increasing level from level A to level 3. Pre procedure one single dose of injection Ceftriaxone one gram after skin testing were given in all patients. Post procedure we prescribed oral fluoroquinolone along with analgesic and alfa blocker to all patients for one week. Patients were followed in OPD after 3 weeks and advised accordingly. Residual stone burden on first follow up was assessed by using X-ray KUB, compared with pre procedural $\mathrm{X}$ ray, if found residual then advised for second session ESWL. If residual stone / un-fragmented stone were found even after three session of ESWL, then we tagged them as ESWL resistant stone. All patients were advised to attend emergency in case of severe colic, hematuria and fever after the procedure. Stone clearance i.e. outcome assessed after 3 months of last session with $\mathrm{X}$ ray KUB, USG KUB and NCCT KUB.

Stone free rate were compared by using student's t-test. Result was considered significant statistically if p-value was less than 0.05 .

\section{RESULTS}

130 patients were screened and 94 were found eligible for randomization as shown in figure-1. The average age of patients in standard group was $38 \pm 1.7$ years and for diuretic group was $36 \pm 1.4$ years. Baseline characteristics of both groups were comparable as shown in Table-1.

Total number of sessions and total number of shocks required to clear the stone is shown in Table-2. 


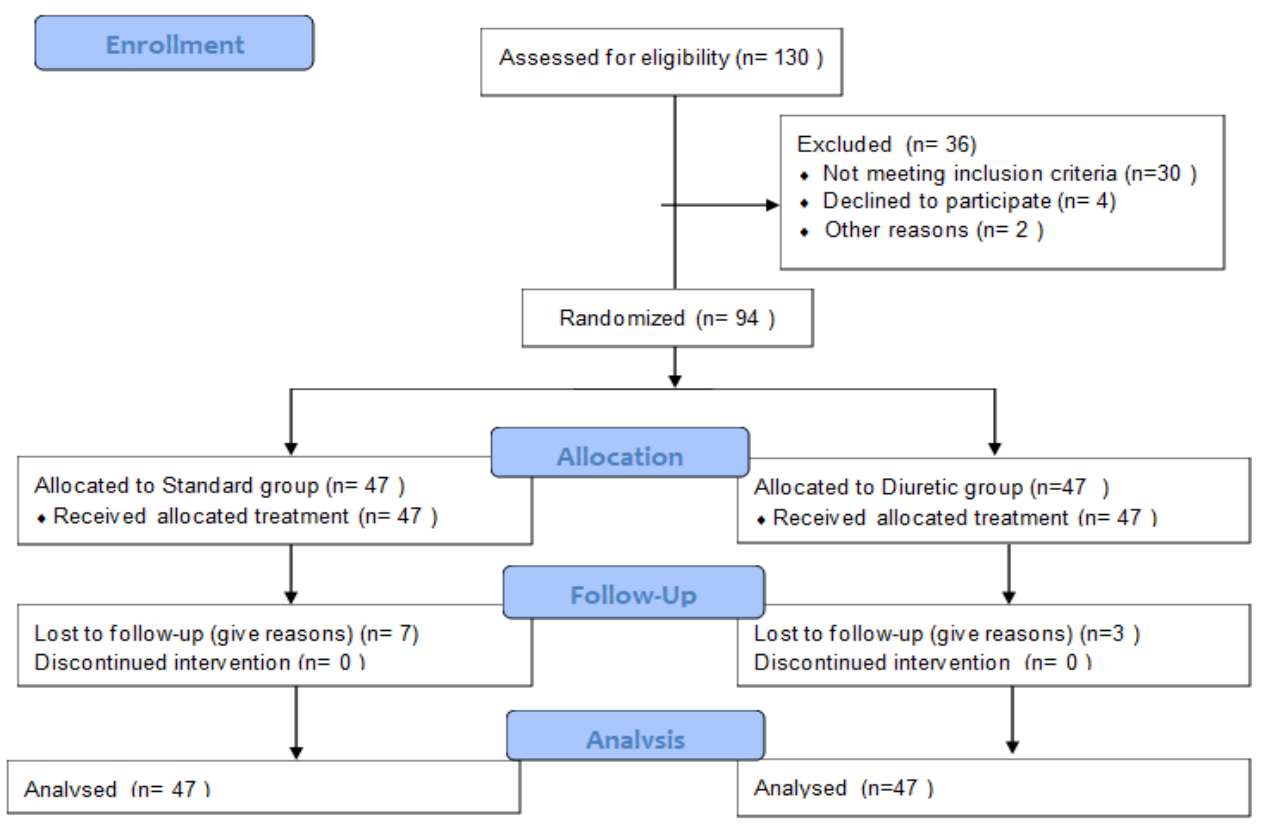

Fig-1: Patient recruitment and follow up chart

Table-1: Baseline clinical, laboratory and imaging findings in the standard and diuretics groups

\begin{tabular}{|l|l|l|l|l|}
\hline & Diuretic Group & Standard Group & Total & p value \\
\hline Age group & & & & \\
$30-40$ & 24 & 28 & 52 & \\
$40-50$ & 16 & 14 & 30 & \\
$50-60$ & 7 & 5 & 12 & \\
\hline Sex & 38 & 41 & 79 & \\
Male & 9 & 6 & 15 & \\
Female & $23.4 \pm 1.2$ & $25.3 \pm 1.1$ & & \\
\hline Body mass index & & & & \\
\hline Side of renal stone & 29 & 25 & 54 & \\
Rt. & 18 & 22 & 40 & \\
Left & $1.21 \pm 0.3$ & $1.26 \pm 0.2$ & & 0.36 \\
\hline Mean stone size (in cm) & $1.12 \pm 0.20$ & $1.21 \pm 0.18$ & & 0.87 \\
\hline Mean Serum Creatinine (mg/dl) & $8.7 \pm 1.2$ & $8.5 \pm 1.6$ & & 0.56 \\
\hline Mean skin stone distance (in cm) & $778 \mathrm{HU} \pm 23$ & $724 \mathrm{HU} \pm 19$ & & 0.09 \\
\hline Mean stone density & & & \\
\hline
\end{tabular}

Table-2: Stone clearance after 3 months with total required sessions and shocks

\begin{tabular}{|l|l|l|l|}
\hline & Diuretic Group & Standard Group & p-value \\
\hline Stone clearance achieved & $39(82.97 \%)$ & $28(59.57 \%)$ & 0.012 \\
\hline Total number of shocks, mean & $4165 \pm 165$ & $4320 \pm 142$ & 0.63 \\
\hline Total number of sessions, mean & $2.2 \pm 0.4$ & $2.3 \pm 0.6$ & 0.74 \\
\hline
\end{tabular}

In our present study, the stone clearance rate was significantly better in diuretic group as compared to standard group $(82.97 \%$ vs $59.57 \% \mathrm{P}<0.05)$.

In standard group 7 patients did not turn up for follow up investigations after 3 months to know the status of residual fragments. 8 patients have remaining fragments in their pelvicalyceal system after 3 months. 4 patients from standard group develop steinstrasse, and they opted laser URSL+ RIRS to get clearance of stone. We considered all 19 patients as failure of ESWL in standard group.
2 patients from diuretic group developed steinstrasse which resolved by conservative with the help of alpha blocker and pain killer. 3 patients did not turn up for follow up investigations after 3 months. 5 patients from this group did not achieve stone clearance completely even after 3 sessions and we consider them failure, although stone was fragmented and reduced in size on follow up. These five patients opted retrograde intra-renal surgery (RIRS) later on. Their stone characters showing higher side of HU (more than 800) and size was also more than $1.3 \mathrm{~cm}$. Follow up was available for all other patients. 


\section{DiSCUSSION}

The procedure of ESWL is effective for stone fragmentation and is a standard procedure for stone size less than $2 \mathrm{~cm}$

Fluid film surrounding the stone reportedly enables improved fragmentation of the stone in ESWL[5]. As mentioned above patients with renal stone are usually in a state of relative dehydration. The dehydration causes relative oliguria which may have a negative effect on stone fragmentation and by compromising the fluid film around the stone Also, if the treated kidney's function has deteriorated due to the obstruction, the dehydration hinders adequate urine flow around the stone even if patient has normal serum creatinine [6]. Diuretics will increase urine flow and thus the probability of cavitation $[7,8]$; which means, pieces of the broken stone shell increase, and the centre of the stone is exposed to subsequent shockwaves allowing entry of urine through the broken surface, thus giving an adequate interface with the centre of the stone [9-11].

The clearance of stone after ESWL depends on many factors such as stone size, location, HU, skin stone distance, intensity of shock, experience of technician/doctor localization of stone etc. Diuretics cause pelvicalyceal fullness and hence it might help in clearance of fragmented stone particles / debris.

In our present study, the stone clearance rate was significantly better in diuretic group as compared to standard group $(82.97 \%$ vs $59.57 \% \mathrm{P}<0.05)$. Our study strictly focused on the effect of diuretic on renal stone of comparable size and location (less than $2 \mathrm{~cm}$ stone in upper and mid pole of either kidney).

Use of diuretics to augment stone fragmentation and clearance has been described earlier by different researchers for ureteric stones and renal stones.

A clinical trial of 106 patients by Axm TA et al., from Egypt [12] compared the effect of infusion of $500 \mathrm{~mL}$ saline containing $40 \mathrm{mg}$ furosemide with that of standard ESWL for the treatment of ureteric stones at different levels. The study reported that the treatment group required fewer sessions and fewer shockwaves/stone, and fragmentation and success rates were better regardless of the stone's location. The calculus fragmentation and 3-month SFR were 93.3\% and $87.5 \%$, respectively for ESWL with diuresis compared to $70.6 \%$ (for fragmentation) for ESWL without diuresis. This study was focused on ureteric stone only with use of diuretics during ESWL.

The influence of diuretic therapy on the success rate of ESWL was also investigated by Zomorrodi et al., 13] The standard ESWL protocol was used in a group of 43 patients, and another group of 43 patients received $40 \mathrm{mg}$ furosemide before ESWL. Regardless of the location of the ureteric stones, they reported that the addition of the diuretic to ESWL therapy improved both stone fragmentation and stone clearance. This study was also focused for ureteric stone only.

In another clinical trial, including 115 patients conducted by Jafri et al., [14], the effect of furosemide on the success rate of ESWL in patients with renal or ureteric stones was studied. The treatment group receiving diuretic had a stone free rate (SFR) of $71.9 \%$ compared with $39.7 \%$ amongst the controls $(\mathrm{P}=0.007)$. Whereas the success rate for those with renal stones was $63.3 \%$ in the treatment group and $43.8 \%$ in the controls, those with ureteric stones had a success rate of $81.5 \%$ vs $20 \%$. The increase in stone clearance rate found in the furosemide treated patients was seen particularly when their BMI was $>30 \mathrm{~kg} / \mathrm{m} 2(81.3 \%$ vs $38.9 \%$ ). In our study the BMI was comparable in both the groups and none had BMI more than 30 . This could be due to exclusion of patients whose skin to stone distance was more than $10 \mathrm{~cm}$ in our study.

Another study investigated the effects of diuretic therapy on renal stones treated with ESWL [15]. The study included 52 patients with renal stones $<2 \mathrm{~cm}$ in diameter, randomized into two groups of 26 , one group was given hydrochlorothiazide twice daily and the other group was given a placebo. This study showed that the diuretic regimen did not affect the SFR after 3 months. This study included oral therapy for diuresis. Hydrochlorthiazide is a weaker diuretic than frusemide and the brisk diuresis within a short duration achievable with frusemide cannot be achieved with hydrochlorthiazide. This may have been one of factors that diminished the beneficial effect of diuretics in stone clearance.

The strength of this study is that it is a prospective randomized study, done under routine healthcare system with same technician. The limitation is its small sample size and small duration of follow up. However, three months is adequate for assessing stone clearance. The relation between position of stone and chances of clearance cannot be commented upon.

\section{Conclusion}

We conclude that there is a beneficial role of intravenous frusemide with hydration versus hydration alone in renal stone fragmentation and clearance by ESWL for solitary stones less than $1.5 \mathrm{~cm}$.

Acknowledgement: I thank Mr. Kumar Pranav, ESWL-Technician for helping me during the whole session of ESWL.

Conflict of interest: None to declare. 


\section{REFERENCES}

1. Alelign T, Petros B. Kidney stone disease: an update on current concepts. Advances in urology. 2018 Feb 4; 2018.

2. Ansari MS, Gupta NP, Hemal AK, Dogra PN, Seth A, Aron M, Singh TP. Spectrum of stone composition: structural analysis of 1050 upper urinary tract calculi from northern India. International journal of urology. 2005 Jan; 12(1):12-6.

3. Harraz AM, Shokeir AA. Urolithiasis in Renal Transplant Donors and Recipients. In Urolithiasis. 2012 (pp. 601-610). Springer, London.

4. Kocaaslan R, Kucukpolat S, Karadag MA. The combination of furosemide and intravenous hydration does not affect the success of shock wave lithotripsy in renal stone treatment: a prospective randomised single blinded trial. Eur $\mathbf{J}$ Hosp Pharm. 2015;22:353-357.

5. Kocaaslan R, Kucukpolat S, Karadag MA, Erkan E, Kadihasanoglu M, Ozyalvacli ME, Toktas MG. The combination of furosemide and intravenous hydration does not affect the success of shock wave lithotripsy in renal stone treatment: a prospective randomised single blinded trial. European Journal of Hospital Pharmacy. 2015 Nov 1;22(6):353-7.

6. Alelign T, Petros B. Kidney stone disease: an update on current concepts. Adv Urol. 2018; 2018:3068365.

7. Ekundayo OJ, Markland A, Lefante C, Sui X, Goode PS, Allman RM, Ali M, Wahle C, Thornton PL, Ahmed A. Association of diuretic use and overactive bladder syndrome in older adults: a propensity score analysis. Archives of gerontology and geriatrics. 2009 Jul 1;49(1):64-8.

8. Liu LR, Li QJ, Wei Q, Liu ZH, Xu Y. Percussion, diuresis, and inversion therapy for the passage of lower pole kidney stones following shock wave lithotripsy. Cochrane Database of Systematic Reviews. 2013(12).

9. Wazir BG, ul Haq MI, ul Haq F, Nawaz A, Nawaz A, Jamil M. Experience of extracorporeal shockwave lithotripsy for kidney and upper ureteric stones by electromagnetic lithotriptor. Journal of Ayub Medical College Abbottabad. 2010 Jun 1;22(2):20-2.

10. Butt AU, Khurram M, Ahmed A. Extracorporeal shock wave lithotripsy. J Coll Physicians Surg Pak. 2005; 15:638-641.

11. Khalique A, Arshad S, Kumar P, Hussain M. Frequency of stone clearance after extracorporeal shockwave lithotripsy for renal stones in adult patients with renal insufficiency. African Journal of Urology. 2017;23(4): 219-223.

12. Azm TA, Higazy H. Effect of diuresis on extracorporeal shockwave lithotripsy treatment of ureteric calculi. Scand J Urol Nephrol. 2002;36:209-212.

13. Zomorrodi A, Golivandan J, Samady J. Effect of diuretics on ureteral stone therapy with extracorporeal shock wave lithotripsy. Saudi Journal of Kidney Diseases and Transplantation. 2008 May 1;19(3):397-400

14. Jafri S, Middour T, Ogan K, Pattaras J. 1894 Comparison Of The Use Of Intraoperative Furosemide On Stone Clearance Rates Wiht Shock Wave Lithotripsy. The Journal of Urology. 2010 Apr;183(4S):e736.

15. Tehranchi A, Rezaei Y, Mohammadi-Fallah M, Mokhtari M, Alizadeh M, Abedi F, Khalilzadeh M, Tehranchi P. Effects of hydrochlorothiazide on kidney stone therapy with extracorporeal shock wave lithotripsy. Urology annals. 2014 Jul;6(3):208-211. 